\title{
Mujer, Gobierno Corporativo eficiencia económicar análisis para el caso de las empresas familiares agroalimentarias
}

\author{
Cinthia Nájera-Vázquez \\ María J. Martínez-Romero
}

RESUMEN: En la actualidad, el estudio de la diversidad de género en el ámbito empresarial está alcanzando una relevancia considerable, debido a la incorporación de la mujer a la vida laboral. Las Empresas Familiares han sido claves para la incorporación de las mujeres al mercado de trabajo, debido, entre otros motivos a la existencia de roles compartidos y al solapamiento de valores familiares y empresariales. La mayor parte de los estudios existentes sobre temas de género se centran en el efecto de la presencia femenina sobre distintas medidas de rendimiento, pero los resultados no son concluyentes en absoluto. Para ahondar en estas cuestiones, el presente trabajo analiza cómo influye la participación femenina en tres órganos de gobierno corporativo: Consejo de Administración, Junta General de Accionistas y Equipo Directivo, sobre la eficiencia económica de las Empresas Familiares Agroalimentarias. Los resultados obtenidos de una muestra de 147 Empresas Familiares Agroalimentarias Españolas, ponen de manifiesto que, la presencia femenina en los Consejos de Administración y en la Junta General de Accionistas, ejerce un impacto positivo y significativo en la eficiencia económica.

PALABRAS CLAVE: Empresa familiar, diversidad de género, gobierno corporativo, eficiencia económica, industria agroalimentaria, consejo de administración, junta general de accionistas, equipo directivo.

CLAVES ECONLIT: G34, L21, L22, 013.

Cómo citar este artículo / How to cite this article: NÁJERA-VÁZQUEZ, C. \& MARTíNEZ-ROMERO, M.J. (2020): "Mujer, Gobierno Corporativo y eficiencia económica: análisis para el caso de las empresas familiares agroalimentarias", CIRIEC-España, Revista de Economía Pública, Social y Cooperativa, 99, 147-176. DOI: 107203/CIRIEC-E.99.15965.

Correspondencia: Cinthia Nájera-Vázquez, E-mail: cinthia_nv4@hotmail.com. ORCID: 0000-0002-98178418; María J. Martínez-Romero, E-mail: mariaj.martinez@ual.es, ORCID: 0000-0001-7891-1558. Universidad de Almería, Ctra. Sacramento s/n, 04120, La Cañada de San Urbano, Almería. Spain. 


\section{EXPANDED ABSTRACT}

\section{Women, Corporate Governance and Economic Efficiency: analysis for the case of Agri-food Family Firms}

\section{Objectives}

Nowadays, the study of gender diversity in the business environment is becoming considerably relevant, due to the incorporation of women into the working life. Social advances have enabled women to leave behind their limited roles at home, and their inclusion in the labour market has gone beyond the compliance with ethics and legislation.

Family firms have played a key role in the inclusion of women in the workplace, due to, among other reasons, the existence of shared roles and the overlapping of family and business values. However, and surprisingly, there are not many studies investigating the role of women in family firms.

In recent years, the academic interest has been focused on gender diversity, namely on the inclusion of women on boards of directors and in top management positions. Specifically, the latest research analyses how gender diversity on boards of directors and in top management positions affects different measures of firm performance, focusing on profitability measurements. However, the obtained results so far are not conclusive at all, revealing that the presence of women on corporate governance bodies can have a positive, a negative or even a non-significant influence on firm performance.

Therefore, the purpose of this paper is shedding some light on this controversial topic, by analysing whether the presence of women in three corporate governance bodies, i.e. the board of directors, the general meeting of shareholders and the top management team, of agri-food family firms influences the firms' economic efficiency.

\section{Methods}

To develop the empirical part of this study, a sample of 147 family firms pertaining to the agri-food industry has been used. The final sample was obtained from a wider study analysing the general characteristics of a representative sample of Spanish family firms. In this regard, the data presented in this article come from two different sources: on the one hand, the financial and economic data were obtained from the SABI (Sistema de Análisis de Balances Ibéricos) database of Bureau Van Dijk; on the other hand, the qualitative data were obtained from the database provided by the Spanish Institute of Family Businesses and the Spanish Network of Family Business Chairs, based on a survey conducted in 2016. The proposed hypotheses were checked by performing ordinary least-squares regression analysis. 


\section{Findings}

The obtained findings suggest that the presence of women on the boards of directors of Spanish agri-food family firms has a positive and very significant influence on economic efficiency. Moreover, the presence of women at the general meeting of shareholders also has a positive and significant influence on the firms' economic efficiency. However, the presence of women in top management teams does not exert a significant impact on economic efficiency.

\section{Contributions}

The present article offers several contributions to the literature. First, this study is pioneering in considering the role exerted by female directors, shareholders and managers in the economic efficiency of family firms. Although women have exerted a fundamental role within business families, their influence in the firm sphere has been very limited. In this vein, our work answers the call for further research on gender issues in family firms.

Second, this study shows that gender diversity on both the board of directors and the general meeting of shareholders of Agrifood family firms has a positive impact on business efficiency, revealing that women influence economic and business development. In this sense, not only is the presence of women attributed to an increase in economic efficiency, but also to an increase in social performance.

Third, with regard to the agri-food industry, this study makes an important contribution at both the theoretical and the empirical level. Studies related to the agri-food industry are usually experimental studies based on comparisons and evaluations to improve production systems, as well as technical studies to achieve sustainable use of natural resources. However, there are few studies aimed at analysing the management of agri-food family firms, with special attention to the participation of a vulnerable stratum, such as women.

As a fourth contribution, we have a sectored study of family firms, which allows to present more precise findings in an important industry for the society and the economy at a global level, which is the agri-food industry.

\section{Practical Implications}

The present work contributes to give visibility to those women present in the corporate governance bodies of agri-food family firms, since it reveals that female participation favours the economic and financial results of these firms.

Although it is true that previous studies have analysed the effect of female presence in cooperative firms, to the best of the authors' knowledge, there are not studies addressing this issue in agri-food family firms. 
Furthermore, from a legal perspective, this work highlights the level of influence that both the Organic Law 3/2007 of March 22nd 2007, for effective equality between women and men (BOE, 2007), and the Law 35/2011 of October 4th 2011, on shared ownership in agricultural holdings (BOE, 2011), have had on the incorporation of women in ownership and management positions in agricultural holdings.

Therefore, the obtained findings in this study may be an incentive to promote the restructuring of corporate governance bodies of agri-food family firms, increasing the presence of women in them.

\section{Limitations}

The contributions of this study are no free of limitations, which, in turn, may provide opportunities for future research. First, we have focused on the economic efficiency of agri-food family firms. Thus, the obtained findings may not be applicable to other industries. However, from our point of view, it is of utmost interest to evaluate the role of women in agri-food family firms to the extent that in southern Spain the agri-food industry is especially relevant for the regional economy, as well as because the issue of gender diversity in the business environment is acquiring special interest in recent years. Another limitation of our study is that we have considered the female presence in three corporate governance bodies, even though the participation of women in any other corporate body can be beneficial to firms. A final limitation would be that the analysed family firms are located in Spain and thus, it would be necessary to determine whether different findings are obtained in other countries or regions.

\section{Originality}

The study of gender diversity in the business environment has emerged as a fruitful field of research in recent years, but the current knowledge regarding the influence of women on firms' outcomes is still scarce. This paper contributes to the family business literature, both in terms of corporate governance and in terms of gender, by offering some conclusions regarding the presence of women in different corporate governance bodies. Furthermore, this paper provides new research avenues that will provide a better comprehension for scholars and specialists for future investigations in the field.

KEYWORDS: Family firms, gender diversity, corporate governance, economic efficiency, agrifood industry, board of directors, general meeting of shareholders, management team. 


\section{Introducción}

La inclusión de las mujeres al mercado de trabajo en los últimos años ha tenido un gran auge (Reguera-Alvarado, De Fuentes y Laffarga, 2017). Los avances sociales han permitido que las mujeres dejen atrás los limitados roles de mujeres en el hogar (Rodríguez-Zapatero, Rodríguez-Jiménez, y Ramírez-Faz, 2013), y su incorporación a la vida laboral ha ido más allá del cumplimiento de la ética y de las legislaciones (Adler, 2003; Chinchilla, Poelmans, y Leon, 2005).

Las empresas familiares, han jugado un papel clave en la inclusión de mujeres en el ámbito laboral, debido a la existencia de roles compartidos y al solapamiento de valores familiares y empresariales (Martínez-Romero y Rojo-Ramírez, 2016). Sin embargo, y sorprendentemente, no existen demasiados trabajos que investiguen el rol de la mujer en la empresa familiar (Martínez-Jiménez, 2009). Los primeros estudios de género en Empresas Familiares revelaban que las mujeres ejercían un rol limitado, debido, entre otros motivos, a la diferencia de estímulos económicos en comparación con los hombres (Rosenblatt, De Milk, Anderson, y Johnson, 1985), al poco interés y dedicación que las mujeres demostraban en comparación con los de los hombres (Kanter, 1977), y a la incertidumbre de los directivos con respecto a la capacidad de las mujeres para ejercer responsabilidades (Burke, 1997). Por el contrario, recientemente se ha demostrado que las mujeres pueden estar al frente de las empresas y participar en cualquier tipo de estructura empresarial, logrando compatibilizar los diferentes roles sociales, empresariales y personales (Burín, 1987; Echebarria-Miguel y Larrañaga-Sarriegui, 2005).

En los últimos años el interés académico se ha centrado en la diversidad de género y la inclusión femenina en consejos de administración y altos cargos de administración (Deloitte, 2017; Hernández-Ortiz, García-Martí, Martínez-Jiménez, Pedrosa-Ortega, y Ruiz-Jiménez, 2020). Concretamente, las últimas investigaciones analizan cómo afecta la diversidad de género en altos cargos de administación a distintas medidas de rendimiento empresarial (Liu, Wei, y Xie, 2014; Martín-Ugedo y Minguez-Vera, 2014), focalizándose en medidas de rentabilidad (por ejemplo, Campbell y Mínguez-Vera, 2008). No obstante, los resultados obtenidos hasta el momento no son para nada concluyentes, revelando que la presencia femenina puede ejercer una influencia positiva (Benito-Osorio, Jiménez, Díaz-Dávila, y Zazo-Vaquero, 2019), negativa (Ryan y Haslam, 2005) e incluso no significativa (Kochan, Bezrukova, Ely, Jackson, Joshi, Jehn, Leonard, Levine, y Thomas, 2003) en el rendimiento empresarial.

La diversidad de los resultados obtenidos ha hecho que numerosos académicos llamen a la investigación sobre temas de género (Cabrera-Fernández, Martínez-Jiménez, y Hernández-Ortiz, 2016; López-Delgado y Diéguez-Soto, 2018; Hernández-Ortiz et al. 2020) en un intento de resolver si la presencia femenina verdaderamente influye o no en los resultados empresariales. Asimismo, se ha requerido la investigación de esta temática en empresas familiares (Cabrera-Fernández et al., 2016; 
Beltrán-Gómez, Haro-Beas, Bernal-Rodríguez, y Cueva-Bravo, 2019), ya que, en la literatura existente predominan las investigaciones que versan sobre empresas cotizadas, y sometidas por tanto a las regulaciones de género (Palomo-Zurdo, Gutiérrez-Fernández, y Fernández-Torres, 2017).

Atendiendo a las recientes llamadas de investigación (Cabrera-Fernández et al., 2016; López-Delgado y Diéguez-Soto, 2018; Hernández-Ortiz et al. 2020), y en un intento de desenredar los resultados existentes, este artículo plantea como principal objetivo analizar no sólo el efecto de la participación femenina en el consejo de administración, sino también en la junta general de accionistas y en el equipo directivo, sobre la eficiencia económica. De este modo el presente trabajo va más allá de la literatura previa, que mayoritariamente analiza la presencia femenina en los consejos de administración (Harris, 2014; Deloitte, 2017) dejando de lado el estudio de otros órganos de gobierno corporativo, como pueden ser la junta general de accionistas o los equipos directivos. Además, este estudio se centra en la eficiencia económica, entendida como la capacidad relativa de la empresa de obtener determinados outputs dada una cierta cantidad de inputs (Martínez-Romero, Martínez-Alonso, Casado-Belmonte, y Rojo-Ramírez, 2019), como medida de rendimiento, superando a los estudios que se limitan a simples medidas de rentabilidad (Carter, Simkins, y Simpson, 2003; Gordini y Rancati, 2017).

Para alcanzar el objetivo propuesto, se han desarrollado una serie de hipótesis en las que se establece que las empresas familiares con mayor número de mujeres en cualquiera de los tres órganos de gobierno corporativo a estudiar son más eficientes. En este sentido, y para cumplir con el objetivo planteado, se ha utilizado una base de datos de 147 Empresas Familiares pertenecientes al sector agroalimentario. Las hipótesis se han testado utilizando la base de datos SABI para los datos económico-financieros y cuestionarios para los datos cualitativos.

Los resultados obtenidos revelan que la presencia de mujeres en los consejos de administración y en la junta general de accionistas, ejerce un efecto positivo y significativo en la eficiencia económica. Además, los resultados ponen de manifiesto que la presencia femenina en el equipo directivo no ejerce una influencia significativa en la eficiencia económica.

Este trabajo presenta importantes contribuciones. En primer lugar, responde a la llamada de investigación sobre temas de género en la empresa familiar (Cabrera-Fernández et al., 2016; Beltrán-Gómez et al., 2019), siendo pionero en considerar el rol que ejercen las mujeres consejeras, accionistas y directivas, en la eficiencia económica en el ámbito este tipo de empresas. En segundo lugar, se confirma la influencia positiva que ejercen las mujeres en el desarrollo económico y empresarial (Abdullah, Ku-Ismail, y Nachum, 2016; Benito-Osorio et al., 2019). Finalmente, este estudio se centra en el sector agroalimentario desde la perspectiva de la gestión y la administración de empresas (MAPA, 2018), lo cual supone en sí mismo una auténtica contribución. 
El trabajo se organiza como sigue. En el siguiente apartado se hace una revisión de literatura y se plantean las hipótesis. A continuación, se describe la metodología y posteriormente se comentan los resultados obtenidos. El trabajo finaliza con el apartado de discusiones, que engloba contribuciones, implicaciones prácticas, limitaciones y futuras líneas de investigación.

\section{Desarrollo de hipótesis}

\subsection{Presencia femenina en el Consejo de Administración y eficiencia de las Em- presas Familiares}

La formación de un consejo de administración con participación femenina es muy reducida (Gómez-Ansón, 2005; Gómez-Álvarez y Sánchez-Barrios, 2009). La universalidad de culturas, pensamientos, habilidades, experiencias, conocimiento y por supuesto, de género, dará lugar a resultados más eficaces que aquellos obtenidos de manera individual. Por lo que se considera que, los consejos de administración que tengan como miembros, personas de todas las capacidades y cualidades, dispondrán de una mayor agudeza para resolver posibles problemas, tener ideas diferenciadas y creativas y conseguir más rápidamente el éxito de cualquier compañía.

Tyson (2003) y Higgs (2003) mencionan en sus investigaciones que las compañías que tienen en su consejo de administración diversidad de género reflejan la diversidad de los distintos grupos de interés, lo que llevará a las compañías a tener un mejor funcionamiento. Los trabajos de Tyson (2003) y Higgs (2003) coinciden en que se tiene que abordar más sobre los temas de género en los consejos de administración de las empresas, ya que las compañías tienen que buscar ampliar sus perspectivas.

El informe "The Female FTSE Report" en 2004, indicó que, en Reino Unido, las empresas que tenían una mayor proporción de mujeres en el consejo de administración tenían un mayor capital bursátil, incrementando el valor de las empresas en el largo plazo.

En Estados Unidos, la revista Fortune 500, que enlista cada año a las 500 mejores empresas estadounidenses por su volumen de ventas, realizó durante cuatro años (1996-2000) un estudio donde concluyó que las 88 empresas cuyos consejos de administración estaban conformados en su mayoría por mujeres, obtuvieron un $35 \%$ más de rentabilidad económica que las empresas que no tenían presencia femenina en sus consejos. 
En Canadá, se estudió la evolución de las empresas que tenían dos o más mujeres en el consejo de administración (Brown, Brown, y Anastasopoulos, 2002) durante los años 1995 y 2001. El citado estudio pone de manifiesto que los consejos de administración con presencia de mujeres eran más independientes y activos, por lo que las empresas con este tipo de consejos obtenían mejores rentabilidades. Además, el estudio concluyó que las empresas incluyentes de diversidad de género en sus consejos de administración tenían clientes más satisfechos.

Carter et al. (2003), Erhardt, Werbel, y Shrader (2003) y Gordini y Rancati (2017) coinciden en sus resultados, mencionando que existe una relación positiva entre presencia de mujeres en los consejos de administración y rentabilidad empresarial, tanto económica como financiera.

Gómez-Ansón (2005) menciona que la presencia de mujeres en el consejo de administración varía dependiendo del sector de las compañías, ya que las empresas que pertenecen a los sectores de bienes de consumo, servicios financieros e inmobiliarias son, en el caso de España, las que mayor porcentaje de mujeres tienen. Asimismo, también se menciona que las denominadas cajas de ahorro sitúan a España en los últimos lugares a nivel europeo, por no tener diversidad de género en sus consejos de administración.

En el año 2010, Carter, D’Souza, Simkins, y Simpson encontraron una relación positiva entre la participación de las mujeres en el consejo y la rentabilidad económica obtenida por las empresas.

Recientemente, Hernández-Ortiz et al. (2020) mencionan que la inclusión de mujeres en los consejos de administración aporta valor agregado a las empresas. Estas mismas autoras, así como Hernández-Nicolás, Martín-Ugedo, y Minguez-Vera (2016) han revelado que las cooperativas agroalimentarias españolas cuyos consejos tienen presencia femenenina total o mayoritaria, presentan resultados favorables en lo que a rentabilidad económica respecta.

Reguera-Alvarado et al. (2017) ponen de manifiesto que la diversidad de género ha ido creciendo en los consejos de administración porque conduce a un buen gobierno corporativo y por tanto, ejerce un efecto positivo en el desempeño empresarial.

Palomo-Zurdo et al. (2017) han concluido que la mayor diversidad de género en el consejo de administración de las cooperativas de crédito españolas tiene una relación directa con la rentabilidad económica de las mismas.

Caso contrario a los estudios anteriores es la investigación de Shrader, Blackburn, y lles (1997) que encuentran una relación negativa entre el porcentaje de mujeres consejeras y la rentabilidad económica y financiera de las compañías para una muestra de 200 sociedades que forman parte de Fortune 500. Del mismo modo, el estudio de Joecks, Pull, y Vetter (2013) también revela su desacuerdo en lo que respecta al impacto positivo en la rentabilidad de las empresas por el hecho de contar con mujeres en los consejos de administración (Joecks et al., 2013). 
Así pues, con la revisión de literatura realizada y en base a los resultados previos, hemos definido la primera hipótesis de nuestro estudio:

H1: Una mayor presencia femenina en el consejo de administración implica una mayor eficiencia económica.

\subsection{Presencia femenina en la Junta General de Accionistas y eficiencia de las Empresas Familiares}

Las empresas familiares se originan generalmente por dos personas unidas emocionalmente, sin tener relación de consanguineidad, que posteriormente dejan a sus herederos, biológicos o no, en las siguientes generaciones (Carsrud, 2006; Karra, Tracey, y Phillips, 2006). Al mencionar a dos personas como iniciadores de la empresa familiar, en la mayoría de los casos, la presencia de las mujeres en el emprendimiento de la empresa familiar ya representa el $50 \%$ de los miembros de la empresa.

Ortiz y Olaz (2014) han encontrado que las empresas familiares que son lideradas por mujeres en la junta de accionistas tienen un consejo de administración donde el $71,4 \%$ de los integrantes son miembros de la familia, frente al $56 \%$ de miembros familiares si la junta de accionistas es liderada por hombres. Esto pone de manifiesto que las mujeres aun cuando tienen una responsabilidad como accionistas mayoritarias, su instinto protector sigue siendo dinámico.

Algunos autores han concluido que la participación de las mujeres en la propiedad de las empresas familiares les ha permitido una visibilidad en los procesos claves del desarrollo de la empresa, ya que han logrado ser reconocidas por el doble rol que realizan (Vera y Dean, 2005; Vadnjal y Zupan, 2009). Sin embargo, años atrás, Dumas (1989) mencionaba que existían barreras para que una mujer pudiera tener el control de la empresa familiar mediante la propiedad, ya que sólo podía tener propiedad por sucesión y no por ser ella la iniciadora de la empresa familiar.

Además, en la literatura se menciona que las mujeres realizan más cuestiones de manera natural, lo que ocasiona que cuando pertenecen a la junta general de accionistas, realicen preguntas que ayudan a procesar y resolver problemas de manera más parcial y transparente (Konrad, Kramer, y Erkut, 2008).

Joy, Carter, Wagner, y Narayanan (2007) mencionan que las empresas con presencia femenina en la junta general de accionistas están mejor organizadas y por ende obtienen mejores resultados que las que no cuentan con mujeres en las juntas, ya que las mujeres realizan prácticas éticas y tienen mayor responsabilidad social corporativa. 
En la década de los $90^{\prime}$ ya se concluía que las mujeres son más exigentes que los hombres para no tener riesgos empresariales, lo que ayuda a hacer frente a los beneficios financieros (Jianakoplos y Bernasek, 1998).

En la actualidad, la empresa más antigua del mundo dedicada a la hostelería y originaria de Japón, ha decidido que la siguiente accionista mayoritaria y directora general será una mujer, lo que pone visible que la presencia de las mujeres como accionistas es la continuidad del éxito de las empresas familiares (Schumann, 2014).

De otro lado, el presidente de una reconocida empresa familiar de la India menciona que el género en la propiedad o en los accionistas mayoritarios no tiene relevancia para el éxito de la empresa, ya que cualquiera que esté al frente de la empresa debe estar calificado para cumplir con sus obligaciones (Philip y Bhattacharyya, 2018).

Según un estudio realizado en Bogotá, Colombia (Berdugo Cotera y Gámez Gutiérrez, 2016), las empresas propiedad de mujeres son pequeñas empresas, de baja inversión e innovación, sugiriendo poner en marcha acciones para el mejoramiento de la productividad y competitividad.

Por tanto, basándonos en la literatura previa, hemos determinado en la segunda hipótesis del trabajo:

H2: Una mayor presencia femenina en la junta general de accionistas implica una mayor eficiencia económica.

\subsection{Presencia femenina en el Equipo Directivo y eficiencia de las Empresas Fa- miliares}

Las empresas familiares incluyen a las mujeres de la familia (madre, esposa, hija, hermana, sobrina, etc.) en algunas de las tareas de gestión, siendo esta una particularidad de este tipo de compañías. Para las mujeres que trabajan en la empresa familiar, existe un desequilibrio entre la vida personal y la empresarial, ya que en algunos casos no reciben remuneración por el trabajo realizado (Rodríguez-Zapatero y Rodríguez-Jiménez, 2011). Una de las desventajas es que en la empresa familiar no se delimitan los horarios y no cabe la posibilidad de optar por una reducción de jornada, además que, las mujeres que son parte de la empresa familiar sienten más responsabilidad, no solo por la parte laboral, sino de mantener una la buena relación entre los miembros de la familia (Danes, Haberman, y McTavish, 2005).

A pesar de las dificultades sociales y personales, en los últimos años, concretamente desde 2011 hasta 2015 , la participación de las mujeres en los equipos directivos ha aumentado un $2 \%$, aunque 
este sigue siendo un incremento bajo. Pavan, Ramachandran, Vishwanathan, y Chittoor (2018) mencionan que las mujeres directivas de empresas familiares en la India han aumentado de un 4,6\% a $13,7 \%$ de 2013 a 2017 , respectivamente.

En 2003 Adler, realizó un estudio de 215 empresas que pertenecían a la revista Fortune 500 en Estados Unidos, y obtuvo que las empresas que incluían a mujeres en sus equipos directivos eran más rentables que la media.

También en América, el estudio publicado en Catalyst (2004), en su investigación obtuvo que las empresas con mayor proporción de mujeres en los equipos directivos obtenían una mayor eficiencia económica.

Se ha visto una relación positiva entre el avance de generaciones de la empresa familiar con la inclusión de las mujeres en equipos directivos, esto quiere decir que las mujeres son opciones para la sucesión y continuidad de las empresas familiares (Dumas, 1998; Ortiz y Olaz, 2014).

Byron y Post (2016) ponen de manifiesto que una alta representación de mujeres en la dirección de las empresas está positivamente relacionada con el desempeño social corporativo. Así como también, en 2012, Adams y Funk realizaron una encuesta a las directivas de las empresas y concluyeron que al ser más benevolentes e inclusivas que sus contrapartes masculinas, las empresas gestionadas por mujeres tiene resultados positivos sociales y, a su vez, económicos.

Además, también se ha puesto de manifiesto que cuando las mujeres dirigen las empresas familiares, hay una mayor presencia tanto de otras mujeres como de otros miembros de la familia (Ortiz y Olaz, 2014). Este hecho demuestra que las mujeres son más incluyentes de personas de su mismo género y que también piensan en el bienestar de los otros miembros de la familia, consiguiendo un avance en la economía y estabilidad social de las regiones (Velandia y Herrera, 2015). El estudio realizado en 2013 por INFORMA, muestra que los equipos directivos con más del 40\% de mujeres tienen menor riesgo de crédito que las empresas que tienen menos del $40 \%$ de mujeres en el equipo directivo.

En el 2008, según el Instituto Nacional de las Mujeres en México (INMUJERES, 2009), las mujeres eran las empleadoras de la quinta parte de todo el país, lo que reflejaba que el sector empresarial estaba liderado por empresas en cuyos equipos directivos había presencia femenina y los resultados financieros eran positivos.

En España, grandes empresas tienen como objetivos a corto plazo, incluir a mujeres en sus equipos directivos y en todos los ejes de gestión, ya que la eficiencia que otras empresas han reflejado ha sido positiva (ISOTES, 2014). 
En un estudio muy reciente (Arioglu, 2020), se demostró que la presencia y el porcentaje de mujeres en el consejo directivo tiene un efecto positivo en el desempeño financiero de las empresas. Del mismo modo, Joecks et al. (2013) manifestaron un efecto positivo en el rendimiento empresarial cuando aumenta la presencia femenina en los equipos directivos. Así mismo, Dezs y Ross (2011) encontraron que, incrementando el número de mujeres en el nivel directivo, se obtenía un mejor desempeño económico.

Bilimoria (2000) menciona que las empresas con presencia femenina en los puestos directivos realzan el valor que las empresas otorgan a las mujeres y reconocen los éxitos logrados por ellas; incrementando con ello, su reputación corporativa en la sociedad.

Las mujeres en la actualidad buscan eliminar las barreras sociales que durante siglos han existido, por lo que han realizado actividades profesionales y personales a la vez, para con ello demostrar la eficacia del género. Esto puede justificar que las mujeres cuando son elegidas para ocupar puestos directivos no quieren ser señaladas con las mismas barreras sociales que los antepasados señalaban, realizando el trabajo con responsabilidad, intentando obtener mejores resultados que los hombres en el mismo puesto directivo.

Una vez analizada la literatura, hemos propuesto una tercera hipótesis para este trabajo:

H3: A mayor presencia femenina en el Equipo Directivo, mayor eficiencia económica.

\section{Metodología}

\subsection{Datos de la muestra}

Para la realización de este trabajo se ha utilizado una muestra de 147 empresas con sede en España consideradas Empresas Familiares pertenecientes al sector agroalimentario. La muestra final fue obtenida de un estudio previo realizado por el Instituto de la Empresa Familiar de España y la Red de Cátedras de Empresa Familiar, a partir de una encuesta realizada en el 2016, que culminó con el desarrollo del libro Factores de competitividad y análisis financiero en la Empresa Familiar (IEF, 2018). Tal y como se pone de manifiesto en el citado estudio, la muestra es representativa de la población de empresas familiares españolas en lo que a su distribución por tamaño empresarial se refiere, predominando las microempresas $(30,1 \%$ ) y las pequeñas empresas $(60,3 \%)$. Asimismo, la muestra también es representativa de la distribución sectorial de las empresas familiares españolas, estando integrada mayoritariamente por empresas de los sectores comercio al por mayor $(31 \%)$ e industria manufacturera $(21,3 \%)$ 
Debido a que el objetivo último del presente trabajo es analizar cómo influye la presencia femenina en los órganos de gobierno de las Empresas Familiares del sector agroalimentario en la eficiencia de las mismas, se han seleccionado de la muestra inicial únicamente las empresas focalizadas en el sector agroalimentario, en cualquiera de las etapas (producción, transformación, comercialización). En este sentido, de las 1005 empresas que conformaban la muestra inicial, un total de 156 pertenecían al sector agroalimentario. Después de eliminar aquellas empresas con valores perdidos respecto de las principales variables objeto de estudio, nuestra muestra quedó configurada por 147 empresas agroalimentarias.

Los datos empíricos presentados en este trabajo provienen de dos fuentes diferenciadas. Por una parte, los datos económico-financieros de las empresas se han obtenido de la base de datos SABI (Sistema de Análisis de Balances Ibéricos) de Bureau Van Dijk para el año 2016 (año en el que se realizó la encuesta). De otro lado, los datos cualitativos se obtuvieron de la base de datos facilitada por el Instituto de la Empresa Familiar y la Red de Cátedras de Empresa Familiar. En este sentido, es importante señalar que el hecho de combinar datos de dos fuentes alternativas mitiga el riesgo de sesgo del método común, ya que la variable dependiente (eficiencia) y las variables de control (tamaño de la empresa, edad de la empresa y rentabilidad económica) proceden de una base de datos externa a la encuesta.

El tratamiento de los datos cualitativos fue llevado a cabo por la empresa NEXO. Las encuestas se realizaron a través de entrevistas telefónicas asistidas por ordenadores (CATI) mediante cuestionario estructurado a CEOs, directivos o responsables de las empresas. También se contó con entrevistas vía correo electrónico para las empresas que lo exigían. La ratio de respuesta obtenido fue de $11,93 \%$ y el error muestral de la encuesta fue de $+/-3,08 \%$ ( $P=Q=50 \%$ y alfa $=0,05)$.

\subsection{Modelo}

Debido a que nuestro objetivo último consiste en determinar el impacto que la presencia femenina en el consejo de administración, en la junta general de accionistas y en el equipo directivo de la empresa, respectivamente, tiene sobre la eficiencia económica empresarial, planteamos los siguientes modelos de regresión, cuyas variables se explican a continuación:

$$
\begin{aligned}
\text { Eficiencia }= & \beta_{0}+\beta_{1} \text { Tamaño }+\beta_{2} \text { Edad }+\beta_{3} \text { REaT }+ \\
& \beta_{4} \text { Presencia Femenina en el Consejo de Administración } \\
\text { Eficiencia }= & \beta_{0}+\beta_{1} \text { Tamaño }+\beta_{2} \text { Edad }+\beta_{3} \text { REaT }+ \\
& \beta_{4} \text { Presencia Femenina en la Junta General de Accionistas } \\
\text { Eficiencia }= & \beta_{0}+\beta_{1} \text { Tamaño }+\beta_{2} \text { Edad }+\beta_{3} \text { REaT }+ \\
& \beta_{4} \text { Presencia Femenina en el Equipo Directivo }
\end{aligned}
$$




\subsection{Variable dependiente}

La variable dependiente del presente estudio, tal y como se especifica en los modelos de regresión anteriores, es la eficiencia económica.

Desde un punto de vista económico-financiero, se define eficiencia, como la capacidad relativa de la empresa de obtener determinados outputs, dada una cierta cantidad de inputs (Martínez-Romero et al., 2019). En este sentido, y basándonos en literatura previa (Leach, Smith, Hart, Morris, Ainsworth, Beterlsen, Iraqui, y Pasari, 1990; Smith, Smith, y Verner, 2006; Erhardt et al., 2003; Martínez-Romero, Rojo-Ramírez, y Casado-Belmonte, 2019), hemos medido la eficiencia económica utilizando el margen de explotación como sigue:

$$
\text { Eficiencia económica }=\frac{\text { Resultado de Explotación }}{\text { Cifra Neta de Negocios }}
$$

\subsection{Variables independientes}

Se han determinado tres variables independientes, ya que se pretende testar si la participación femenina, ya sea en el consejo de administración, en la junta de accionistas o en el equipo directivo, afecta o no la eficiencia económica. Por tanto, nuestras variables independientes son:

Presencia Femenina en el consejo de administración $=\frac{\text { Número de Mujeres en el Consejo }}{\text { Número total de miembros en el consejo }}$

Presencia Femenina en la junta general de accionistas $=\frac{\text { Número de Mujeres en la Junta General de Accionistas }}{\text { Número total de accionistas }}$

Presencia Femenina en el equipo directivo $=\frac{\text { Número de Mujeres en el Equipo Directivo }}{\text { Número total de directivos }}$

Para conocer estos datos, en la encuesta se preguntaba directamente por el porcentaje de mujeres en cada uno de los órganos de gobierno corporativo y dirección, respectivamente. 


\subsection{Variables de control}

Además, en este estudio se han incluido las siguientes variables de control:

- Tamaño de la empresa. Medido a través del número de empleados (Hauck y Prügl, 2015; De Massis, Kotlar, Mazzola, Minola, y Sciascia, 2018), ya que se considera que las empresas más grandes son más eficientes debido al aprovechamiento de economías de escala y a un mayor poder de mercado (Cassiman y Veugelers, 2006). Además, estudios previos ponen de manifiesto que el tamaño de la empresa tiene que ver con la inclusión de mujeres en las empresas en los órganos de gobierno corporativo (INFORMA, 2019). Para conseguir una distribución más normal, se ha utilizado el logaritmo neperiano del número de empleados (Tabachnick y Fidell 1996).

- Edad de la empresa. Esta variable se midió como el número de años que lleva la empresa en funcionamiento, desde su fundación hasta el año en el que se realizó la encuesta, esto es, en 2016 (Diéguez-Soto, Manzaneque, y Rojo-Ramírez, 2016; Vandekerkhof, Steijvers, Hendriks, y Voordeckers, 2019). Para esta variable, nuevamente se calculó el logaritmo neperiano con la finalidad de obtener una distribución más normal (Tabachnick y Fidell, 1996). La literatura previa considera que, a mayor edad de la empresa, mayor es su productividad (Ayala y Navarrete, 2004).

- Rentabilidad económica antes de impuestos (REaT). Algunos autores mencionan que la rentabilidad económica de la empresa tiene relación directa con la productividad (Adendorff, Boshoff, Court, y Radloff, 2005; Schumacher, 2015). Así pues, siguiendo a Martínez-Romero y Rojo-Ramírez (2017), se ha calculado la REaT mediante la siguiente expresión:

$$
\mathrm{REaT}=\frac{\mathrm{RBE}}{\mathrm{Cl}}
$$

Donde:

RBE: Resultado Bruto de la Explotación.

Cl: Capital invertido.

En línea con estudios previos (por ejemplo, Cruz, Larraza-Kintana, Garcés-Galdeano, y Berrone, 2014; Martínez-Romero et al., 2019), y para evitar problemas con la transformación logarítmica de los valores negativos de la REaT, añadimos 1 a todos los valores originales de la rentabilidad económica antes de calcular el logaritmo neperiano. 


\section{Análisis de los resultados}

La tabla 1 muestra los estadísticos descriptivos para las variables analizadas, dependiente (eficiencia) e independientes (mujeres en los tres órganos de gobierno corporativo) y control (edad, tamaño y rentabilidad). Se muestra que el valor medio de la eficiencia es 0,065 , habiendo compañías en las que la mínima registrada es de $-0,88$ y la máxima de 0,68 . El tamaño empresarial, representado por el capital invertido de las empresas analizadas mostró una media de 6.860 miles de euros. Por otro lado, la edad media de las empresas analizadas es de 44 años, con un mínimo de 5 y un máximo de 1000 años. La media de la rentabilidad económica de las empresas fue de 0,10. Los valores de los estadísticos descriptivos de las variables independientes se han mostrado en porcentaje. Así, se muestra que, de media, en los consejos de administración, hay un 13,65\% de mujeres, estando el porcentaje de mujeres en los consejos, comprendido entre el cero y el cien por ciento. Además, se puede observar, que el porcentaje de mujeres en la junta general de accionistas de las empresas analizadas está en torno al 8,33\%. Es de destacar que la presencia femenina en la junta general de accionistas toma un valor máximo del $75 \%$, lo cual pone de manifiesto que en el $25 \%$ de las empresas no existen mujeres en la junta general de accionistas. Finalmente, se pone de manifiesto que las mujeres están presentes en los equipos directivos de las empresas analizadas en un porcentaje cercano al 9,44\%, y que este porcentaje oscila entre una representación nula (0\%) y una representación absoluta (100\%).

\section{Tabla 1. Estadísticos descriptivos de las variables}

\begin{tabular}{|l|c|c|c|c|}
\hline \multicolumn{1}{|c|}{ Variables } & Media & Desviación típica & Mínimo & Máximo \\
\hline Eficiencia & 0,068 & 0,13 & $-0,88$ & 0,68 \\
\hline Tamaño Cl & 29.99 & 45,60 & 1 & 381 \\
\hline Edad empresarial & 44,22 & 84,47 & 5,00 & $1.017,00$ \\
\hline REaT & 0,10 & 0,11 & $-0,40$ & 0,47 \\
\hline \% Mujeres Consejo de Administración & 13,65 & 23,07 & 0,00 & 100 \\
\hline \% Mujeres Junta de Accionistas & 8,33 & 17,77 & 0,00 & 75 \\
\hline \% Mujeres Equipo Directivo & 9,44 & 20,06 & 0,00 & 100 \\
\hline $\begin{array}{l}\text { N=147 } \\
\text { REaT= Rentabilidad económica antes de impuestos. }\end{array}$ \\
\hline
\end{tabular}

La tabla 2 muestra las correlaciones de Pearson, que ponen manifiesto las relaciones existentes entre las distintas variables objeto de estudio en este trabajo. Se puede observar que tanto la rentabilidad económica $(p<0,01)$, como la presencia femenina en el consejo de administración $(p<0,05)$ 
y en la junta general de accionistas $(p<0,1)$, están positiva y significativamente correlacionadas con la eficiencia económica.

Además, la matriz de correlaciones no muestra indicios de multicolinealidad, en tanto en cuanto, los coeficientes de correlación entre las variables independientes tienen valores modestos, todos ellos menores del límite de 0,80 por encima del cual los problemas de multicolinealidad pueden surgir (Gujarati y Porter, 2008).

Las hipótesis propuestas han sido testadas utilizando modelos de regresión por mínimos cuadrados ordinarios. Los resultados de la regresión se muestran en la tabla 3, que considera la eficiencia como variable dependiente. El Modelo 1 es el modelo base que incluye únicamente las variables de control. Las variables independientes, se incluyen en los Modelos 2, 3 y 4, respectivamente.

El Modelo 1 muestra que la rentabilidad económica $(\beta=0,747 ; p \leq 0,01)$, ejerce un efecto positivo y altamente significativo sobre la eficiencia de las empresas familiares agroalimentarias. Se puede observar que, el tamaño de las empresas ejerce un efecto positivo, aunque no significativo en la eficiencia, y la edad ejerce un efecto negativo, sobre la eficiencia, aunque tampoco es significativo. Además, el $\mathrm{R}^{2}$ ajustado del modelo es de 0,380 y el modelo es altamente significativo $(p \leq 0,01)$.

El Modelo 2 también es altamente significativo ( $p \leq 0,01)$, el $R^{2}$ ajustado aumenta a 0,404 con la inclusión de la variable presencia femenina en el consejo de administración. Las variables de control se comportan en el Modelo 2 de forma similar a como se comportaban en el primer modelo, en tanto en cuanto que la rentabilidad económica sigue ejerciendo un efecto positivo y significativo sobre la variable dependiente $(\beta=0,760 ; p \leq 0,01)$, y la edad y el tamaño siguen sin ser significativos. Además, la presencia de mujeres en el consejo de administración ejerce una influencia positiva y altamente significativa en la eficiencia económica $(\beta=0,001 ; p \leq 0,01)$. Por tanto, los resultados soportan la hipótesis 1.

El Modelo 3 incluye otra de las variables independientes, en este caso la presencia femenina en la junta general de accionistas, medida como se comentó anteriormente, como el porcentaje de mujeres presente en dicha junta. El modelo sigue siendo altamente significativo $(p \leq 0,01)$. Sin embargo, la $\mathrm{R}^{2}$ ajustada disminuye respecto al Modelo 2 a un valor de 0,388 , pero sigue siendo mayor que la $\mathrm{R}^{2}$ ajustada del Modelo 1 (modelo base). Los resultados de la tabla 3 ponen de manifiesto que el porcentaje de mujeres en la junta de general de accionistas ejerce un impacto positivo y significativo sobre la eficiencia económica $(\beta=0,001 ; p \leq 0,1)$. Los resultados pues, soportan la hipótesis 2 .

En el Modelo 4, que es altamente significativo ( $p \leq 0,01)$, la $\mathrm{R}^{2}$ ajustada es 0,375 y, por tanto, menor que en los Modelos 1, 2 y 3. Sin embargo, en este Modelo se muestra que la presencia de las mujeres en el equipo directivo no ejerce un impacto significativo en la eficiencia de las empresas familiares agroalimentarias. Así, los resultados no soportan la hipótesis 3. 
En conjunto, y tomando en consideración las tres hipótesis planteadas, podemos decir que los resultados verifican las hipótesis 1 y 2 , ya que se demuestra que a medida que se incrementa el porcentaje de mujeres, tanto en el consejo de administración como en la junta general de accionistas, la eficiencia alcanzada por las empresas familiares agroalimentarias es mayor. Por el contrario, los resultados no permiten verificar la hipótesis 3 , por lo que no podemos afirmar que la presencia de las mujeres en el equipo directivo de las empresas familiares agroalimentarias esté relacionada con la eficiencia económica.

\section{Tabla 2. Matriz de correlaciones por pares}

\begin{tabular}{|l|c|c|c|c|c|c|c|}
\hline \multicolumn{1}{|c|}{ Variables } & 1 & 2 & 3 & 4 & 5 & 6 & 7 \\
\hline 1. Eficiencia & 1 & & & & & & \\
\hline 2. Tamaño Cl & 0,130 & 1 & & & & & \\
\hline 3. Edad & $-0,066$ & 0,093 & 1 & & & & \\
\hline 4. REaT & $0,274^{* * *}$ & 0,110 & $-0,059$ & 1 & & & \\
\hline 5. \% Mujeres Consejo de Administración & $0,138^{* *}$ & 0,053 & $-0,011$ & 0,005 & 1 & & \\
\hline 6. \% Mujeres Junta de Accionistas & $0,162^{*}$ & $0,155^{*}$ & $-0,003$ & 0,058 & $0,540^{* * *}$ & 1 & \\
\hline 7. \% Mujeres Equipo Directivo & 0,042 & 0,84 & $-0,059$ & 0,059 & $0,293^{* * *}$ & $0,355^{* * *}$ & 1 \\
\hline $\mathrm{N}=147 .{ }^{*} p \leq 0,10 ;{ }^{* *} p \leq 0,05 ;{ }^{* * *} p \leq 0,01$ & & & & \\
\hline
\end{tabular}

\section{Tabla 3. Análisis de regresión}

\begin{tabular}{|c|c|c|c|c|}
\hline Variables & Modelo 1 & Modelo 2 & Modelo 3 & Modelo 4 \\
\hline Constante & $0,024(0,609)$ & $0,011(0,804)$ & $0,022(0,639)$ & $0,025(0,602)$ \\
\hline Tamaño $^{1}$ & $0,000(0,949)$ & $-0,002(0,811)$ & $-0,002(0,759)$ & $0,000(0,957)$ \\
\hline Edad $^{1}$ & $-0,007(0,565)$ & $-0,007(0,592)$ & $-0,007(0,579)$ & $-0,007(0,561)$ \\
\hline REaT $^{1}$ & $0,747^{\star \star *}(0,000)$ & $0,760^{\star * *}(0,000)$ & $0,740^{* * *}(0,000)$ & $0,748^{\star * *}(0,000)$ \\
\hline \% Mujeres Consejo de Administración & & $0,001^{* \star *}(0,010)$ & & \\
\hline \% Mujeres Junta de Accionistas & & & $0,001^{*}(0,089)$ & \\
\hline \% Mujeres Equipo Directivo & & & & $0,000(0,889)$ \\
\hline$R^{2}$ & 0,392 & 0,420 & 0,405 & 0,392 \\
\hline$R^{2}$ Ajustado & 0,380 & 0,404 & 0,388 & 0,375 \\
\hline $\mathrm{F}$ & $30,786^{\star \star *}$ & $25,747^{\star \star \star}$ & $24,138^{\star \star *}$ & $22,936^{\star \star *}$ \\
\hline \multicolumn{5}{|c|}{$\begin{array}{l}N=147 \text {. Variable dependiente=eficiencia económica. Errores estándar robustos entre paréntesis. } \\
{ }^{1} \text { Logaritmo neperiano utilizado en el análisis de regresión. } \\
{ }^{*} p \leq 0,10 ;{ }^{* *} p \leq 0,05 ;{ }^{* \star} p \leq 0,01 \text {. }\end{array}$} \\
\hline
\end{tabular}




\section{Discusión y conclusiones}

Como se expresó en la introducción, el presente estudio tenía como objetivos principales evaluar la influencia de la presencia de mujeres en tres órganos de gobierno corporativo (consejo de administración, junta general de accionistas y equipo directivo) de empresas familiares agroalimentarias, sobre la eficiencia económica de las mismas.

Para analizar la influencia femenina en la eficiencia económica, se propusieron tres hipótesis, una por cada variable independiente (porcentaje de mujeres en el consejo de administración, en la junta general de accionistas y en el equipo directivo, respectivamente). Las hipótesis fueron testadas con una muestra de 147 empresas familiares españolas, todas ellas dedicadas en el sector agroalimentario en cualquiera de sus etapas de la cadena agroalimentaria, realizando un análisis de regresión por mínimos cuadrados ordinarios. Los resultados mostraron que la presencia femenina en los consejos de administración de las empresas familiares agroalimentarias españolas ejerce una influencia positiva y muy significativa en la eficiencia económica (H1), así como también que la presencia de mujeres en la junta general de accionistas influye positiva y significativamente en la eficiencia de las empresas $(\mathrm{H} 2)$. Sin embargo, pese a tener una relación positiva, no se detectó una relación significativa entre la participación de mujeres en el equipo directivo y la eficiencia de la empresa $(\mathrm{H} 3)$.

Los resultados de las hipótesis 1 van en la misma línea que los resultados obtenidos por Boulouta (2013), Gordini y Rancati (2017) y Cruz, Justo, y Larraza-Kintana (2018) los cuales revelan que, la presencia femenina en los consejos de administración tiene relación directa con una mayor productividad financiera, empresarial y social, respectivamente. Los resultados relativos al porcentaje de mujeres en el consejo de administración (13,65\%), coinciden con los obtenidos por Hernández-Ortiz et al. (2020), que mencionan que las mujeres siguen ocupando papeles secundarios en las sociedades cooperativas agroalimentarias españolas. Del lado opuesto, encontramos un estudio del Corporate Women Directors que mostró que el 76\% de las mayores empresas españolas exitosas financieramente en el ejercicio 2000, no contaban con mujeres en su consejo de administración (Gómez-Álvarez y Sánchez-Barrios, 2009).

Los resultados obtenidos se podrían sustentar en las siguientes razones. Por un lado, el consejo de administración es un órgano en el que pueden participar mujeres que no sean de la misma familia, las cuales, incrementan la diversidad de pensamientos y no influyen en la esfera familiar de la empresa. Mientras que, por otro lado, las mujeres que pertenecen a la familia y al consejo de administración, buscan siempre el éxito de la empresa porque ven en ella una oportunidad para tener éxito económico y profesional, liderando de manera natural. Otro argumento es que, la presencia de mujeres en el 
consejo de administración va en línea con el cumplimiento de la regulación de igualdad de género (Art. 45 de la Ley Orgánica 3/2007 de Igualdad; BOE, 2007), lo cual ha contribuido a favorecer tanto la imagen social de las empresas como su eficiencia.

Los resultados que corroboran la hipótesis 2, pueden obedecer a distintas explicaciones. Por una parte, las mujeres por naturaleza son protectoras, y en este caso, si son propietarias de la empresa, tienden a proteger sus acciones de manera natural hasta conseguir resultados positivos, y con ello sentirse orgullosas de lo que han creado. Por otra parte, la organización de diferentes actividades a la vez es una habilidad femenina, por lo que mujeres propietarias, llevan a la misma vez la organización familiar y la de la empresa, sin diferenciar las responsabilidades de una 0 de otra, siempre buscando los mejores resultados en ambos entornos.

Las empresas familiares agroalimentarias teniendo presencia femenina en la junta general de accionistas, además de influenciar positivamente la eficiencia económica, estarían siendo partícipes de la Ley 35/2011 sobre titularidad compartida en explotaciones agrarias (BOE, 2011), poniendo de manifiesto que cuando las mujeres pasan a tener la titularidad compartida, tienen un mayor compromiso legal con la empresa y por ende, tienen un mayor control económico y social para obtener resultados económico-financieros eficientes.

Actualmente, se han conformado organizaciones 0 asociaciones de mujeres accionistas o propietarias de empresas que se dedican a ayudar, capacitar, formar y fomentar la incorporación formal de mujeres en las empresas, especialmente en las de carácter familiar. Además, existen diversas medidas a nivel europeo consistentes en premios y reconocimientos a mujeres que lideran empresas, lo cual ha motivado a los propietarios hombres a compartir la responsabilidad de las empresas con mujeres (ya sean hijas o esposas). Esta última, puede ser una razón de peso por la cual las mujeres toman decisiones formales y visibles en el ámbito empresarial, que generan más y mejores resultados económico-financieros.

Las mujeres propietarias integran a todos los que pertenecen a la empresa en las diferentes actividades que la misma desarrolla, sean de la familia o no, y con ello se logra una mejor eficiencia, debido a un mayor compromiso empresarial (Ortiz y Olaz, 2014). En este sentido, Dumas (1998) menciona que el liderazgo de las mujeres que son sucesoras en las empresas familiares viene por la implicación social que tienen desde pequeñas y eso les ayuda a tener un compromiso empresarial cuando llega el momento de la sucesión. Asimismo, las mujeres propietarias humanizan dicho proceso de sucesión, según Edlund (1992). Es más, determinados autores establecen que esto tiene que ver con la teoría de la dependencia de los recursos, que postula que las mujeres logran una mejor rentabilidad económica en las empresas a las que pertenecen, debido entre otros motivos, a las donaciones caritativas (Wang y Coffee, 1992). 
Los resultados obtenidos en la hipótesis 3 no fueron los esperados, ya que en algunas investigaciones se muestra una relación positiva y significativa entre la presencia de mujeres en los equipos directivos y la productividad de las empresas familiares (Finkelstein, 1992; Bernardi, Bosco, y Vassill, 2006; Cruz et al., 2018). Aunque en el presente estudio, se obtuvo una relación positiva, no fue corroborada estadísticamente. Los resultados obtenidos pueden deberse al propio sector objeto de estudio, ya que se trata de un sector que en la actualidad y culturalmente aún es mayoritariamente dirigido por hombres, lo que puede influir en el desarrollo de las habilidades de las mujeres que pertenecen al equipo directivo, o bien, al hecho de que las mujeres del equipo estén limitadas en los procesos de toma de decisiones. Por lo tanto, los resultados obtenidos ponen de manifiesto que las mujeres que pertenecen al equipo directivo no ejercen una influencia significativa en la eficiencia económica. En este sentido, se debería considerar el incremento de la diversidad de género en este órgano de gobierno corporativo, para tratar de lograr una mayor eficiencia económica en las empresas familiares agroalimentarias españolas, y así alcanzar niveles similares a los de empresas de otros sectores (Dumas, 1998; Adler, 2003; Ortiz y Olaz, 2014).

\subsection{Contribuciones}

El presente trabajo hace varias contribuciones a la literatura. En primer lugar, este trabajo es uno de los primeros en considerar el rol que ejercen las mujeres consejeras, accionistas y directivas, en la eficiencia económica en el ámbito de la empresa familiar. Aunque, como se comentó previamente, las mujeres han ejercido un papel fundamental dentro de las familias empresarias, su influencia en la esfera empresarial ha estado muy limitada (Schein y Mueller, 1992; Oakley, 2000; Ortiz y Olaz, 2014; MAPA, 2018). En este sentido, nuestro trabajo responde a la llamada sobre investigación en temas de género en la empresa familiar, para lograr una mejor comprensión de la dinámica de este tipo de empresas (MICIU, 2011; Beltrán-Gómez et al., 2019).

En segundo lugar, se ha puesto de manifiesto que la diversidad de género tanto en el consejo de administración como en la junta general de accionistas de las empresas familiares agroalimentarias tiene consecuencias positivas en la eficiencia económica, revelando que las mujeres ejercen una influencia para el desarrollo económico y empresarial (Abdullah et al., 2016; Benito-Osorio et al., 2019). En este sentido, no solo se atribuye a la presencia femenina un incremento de la eficiencia económica, sino también incremento del desempeño social (Arredondo-Trapero, Velázquez-Sánchez, y de la Garza-García, 2013; Cruz et al. 2018).

En tercer lugar y en lo que respecta al sector agroalimentario, este trabajo supone una importante contribución a nivel teórico y empírico. Los trabajos relacionados con el sector agroalimentario son habitualmente estudios experimentales que se basan en hacer comparaciones y evaluaciones para mejorar los sistemas de producción, así como también existen estudios e investigaciones técnicas y económicas para hacer un uso sostenible de los recursos naturales. Sin embargo, existen pocos 
trabajos que vayan encaminados al análisis de la gestión, organización o dirección de las empresas familiares agroalimentarias, y con especial atención a la participación de un estrato vulnerable como son las mujeres.

Como cuarta contribución tenemos, un estudio sectorizado y específico de las empresas familiares, permitiendo dar a conocer resultados más precisos en un sector importante para la sociedad y la economía a nivel mundial, que es el agroalimentario.

\subsection{Implicaciones prácticas}

El presente trabajo contribuye a dar visibilidad a las mujeres presentes en los órganos de gobierno de las empresas familiares agroalimentarias, ya que revela que la participación femenina favorece los resultados económico-financieros de dichas empresas.

Si bien es cierto que, estudios previos han analizado el efecto de la presencia femenina en empresas cooperativas (Esteban-Salvador, Gargallo-Castel y Pérez-Sanz, 2012; Esteban-Salvador, Pérez-Sanz, y Gargallo-Castel, 2017; Hernández-Ortiz et al., 2020), hasta donde el conocimiento de los autores llega, no existen trabajos de investigación que aborden esta cuestión para el caso de empresas familiares agroalimentarias.

Además, desde una perspectiva jurídica, este trabajo pone de manifiesto el nivel de influencia que tanto la Ley Orgánica 3/2007, de 22 de marzo, para la igualdad efectiva de mujeres y hombres (BOE, 2007), como la Ley 35/2011, del 4 de octubre, sobre titularidad compartida en explotaciones agrarias (BOE, 2011), han tenido en la incorporación de la mujer a cargos directivos y de titularidad de las explotaciones agrarias.

Por lo tanto, los resultados obtenidos en el presente estudio pueden ser un aliciente para promover la reestructuración de los órganos de gobierno de las empresas familiares agroalimentarias, incrementando la presencia femenina en los mismos.

\subsection{Limitaciones}

No queremos dejar de mencionar las limitaciones que presenta este trabajo. En primer lugar, nos hemos centrado en la eficiencia de las empresas familiares pertenecientes al sector agroalimentario, por lo que los resultados obtenidos pueden no ser aplicables a otros sectores. Sin embargo, consideramos de interés evaluar el papel de las mujeres en la empresa familiar agroalimentaria debido a que en el sur de España la actividad agroalimentaria es especialmente relevante en la economía regional, así como también, porque el tema de diversidad de género en el ámbito empresarial está adquiriendo un especial interés en los últimos años (Rodríguez-Zapatero y Rodríguez-Jiménez, 2011; 
Rodríguez-Zapatero et al., 2013; Ortiz y Olaz, 2014; Velandia y Herrera, 2015; Vershinina, Rodgers, McAdam, y Clinton, 2019). Otra limitación de este trabajo es que se ha considerado la presencia femenina en tres órganos de gobierno corporativo, aun cuando la participación de mujeres en cualquier otro órgano empresarial puede ser beneficiosa para las empresas. Una última limitación sería que las empresas familiares analizadas están localizadas en España, por lo que habría que determinar si se obtienen diferentes resultados en otros países o en otras regiones.

\subsection{Futuras líneas de investigación}

Como se mencionó anteriormente, en el presente trabajo se ha estudiado la eficiencia económica, por lo que, en el futuro, podría ser interesante analizar otras variables económico-financieras de las empresas familiares agroalimentarias. Así pues, se pueden realizar muchas investigaciones relacionadas al tema, haciendo hincapié en distintos resultados empresariales, ya que este enfoque abre un amplio abanico de posibilidades para el sector agroalimentario, concretamente para lo relativo a la participación de las mujeres en este tipo de empresas. Por ejemplo, estudios similares al que aquí se presenta, pero a nivel internacional o mundial, en áreas donde la agroalimentación es la principal fuente económica, pueden ser líneas futuras de investigación interesantes. Se podría replicar este estudio para otros sectores económicos, así se podría ver la influencia de las mujeres en otras áreas de interés. Todos estos temas podrían ser muy novedosos y aportar más información en el ámbito de las empresas familiares, ya que mezcla una sectorización de las actividades económicas con una parte importante de la sociedad, que es el género.

\section{Bibliografía}

ABDULLAH, S.N., KU-ISMAIL, N.I. \& NACHUM, L. (2016): "Does having women on boards create value? The impact of societal perceptions and corporate governance in emerging markets", Strategic Management Journal, 37, 466-476, DOI: 10.1002/smj.2352, DOI: 10.1002/smj.2352.

ADAMS, R.B. \& FUNK, P. (2012): "Beyond the glass ceiling: Does gender matter?", Management Science, 58, 219-235, DOI: 10.1287/mnsc.1110.1452.

ADENDORFF, C., BOSHOFF, C., COURT, P. \& RADLOFF, S. (2005): "The impact of planning on good governance practice in South African Greek family business", Management Dynamics, 14(4), 3446. 
ADLER, R.D. (2003): Women in the executive suite correlate to high profits, European Project on Equal Pay.

ARIOGLU, E. (2020): "Female board members: the effect of director affiliation", Gender in Management, 35(2), 225-254, DOI: 10.1108/GM-05-2019-0080.

ARREDONDO-TRAPERO, F.G., VELÁZQUEZ-SÁNCHEZ, L.M. \& DE LA GARZA-GARCÍA, J. (2013): "Políticas de diversidad y flexibilidad laboral en el marco de la responsabilidad social empresarial. Un análisis desde la perspectiva de género", Estudios Gerenciales, 29(127), 161-166, DOI: 10.1016/j.estger.2013.05.004.

AYALA, J.C. \& NAVARRETE, E. (2004): "Efectos tamaño y sector sobre la rentabilidad, endeudamiento y coste de la deuda de las empresas familiares riojanas", Cuadernos de Gestión, 4(1), 35-53.

BELTRÁN-GÓMEZ, L.L., HARO-BEAS, J.F., BERNAL-RODRÍGUEZ, F.F. Y CUEVA-BRAVO, O.A. (2019): "Impacto del liderazgo de la mujer en la empresa familiar", EDUCATECONCIENCIA, 22(23), 25-39.

BENITO-OSORIO, D., JIMÉNEZ, A., DÍAZ-DÁVILA, C. \& ZAZO-VAQUERO, M. (2019): "La diversidad de género en los consejos de administración y el rendimiento empresarial: perspectivas actuales y futuras", Management international/International Management/Gestiòn Internacional, 23(2), 1222, DOI: 10.7202/1060027ar.

BERDUGO COTERA, E. \& GÁMEZ GUTIÉRREZ, J. (2016): "Mujeres empresarias de Bogotá", Revista CIFE: Lecturas de Economía Social, 26, 149-173, DOI: 10.15332/s0124-3551.2015.0026.05.

BERNARDI, R.A., BOSCO, S.M. \& VASSILL, K.M. (2006): "Does female representation on boards of directors associate with fortune's 100 'best companies' list?", Business \& Society, 45(2), 235-248, DOI: $10.1177 / 0007650305283332$.

BILIMORIA, D. (2000): Building the business case for women corporate directors, International Challenges and Opportunities, The Netherlands.

BOE (2007): Ley Orgánica 3/2007, de 22 de marzo, para la igualdad efectiva de mujeres y hombres, Agencia Estatal Boletín Oficial del Estado No. 71, 23/03/2007.

BOE (2011): Ley 35/2011, de 4 de octubre, sobre titularidad compartida de las explotaciones agrarias, Boletín Oficial del Estado №. 240, 05/10/11.

BOULOUTA, I. (2013): "Hidden Connections: The Link Between Board Gender Diversity and Corporate Social Performance", Journal of Business Ethics, 113(2), 185-197, DOI: 10.1007/s10551-0121293-7.

BROWN, D.A., BROWN, D.L. \& ANASTASOPOULOS, V. (2002): Woman on boards: Not just the right thing... but the "bright" thing, Conference Board of Canada.

BURÍN, M. (1987): Estudios sobre la subjetividad femenina. Mujeres y Salud Mental. 
BURKE, R. (1997): "Women on corporate boards of directors: a needed resourse", Journal of Business Ethics, 16(9), 909-915, DOI: 10.1023/A:1017987220508.

BYRON, K. \& POST, C. (2016): "Women on Boards of Directors and Corporate Social Performance: A Meta-Analysis", Corporate Governance: An International Review, 24(4), 428-442, DOI: 10.1111/ corg. 12165.

CABRERA-FERNÁNDEZ, A.I, MARTÍNEZ-JIMÉNEZ, R. \& HERNÁNDEZ-ORTIZ, M.J. (2016): "Women's Participation on Boards of Directors: A Review of the Literature", International Journal of Gender and Entrepreneurship, 8(1), 69-89, DOI: 10.1108/JJGE-02-2015-0008.

CAMPBELL, K. \& MINGUEZ-VERA, A. (2008): "Gender diversity in the boardroom and firm financial performance", Journal of Business Ethics, 83(3), 435-451, DOI: 10.1007/s10551-007-9630-y.

CARSRUD, A.L. (2006): "Commentary: Are we family and are we treated as family? Nonfamily employees' perceptions of justice in the family firm: It all depends on perceptions of family, fairness, equity, and justice", Entrepreneurship Theory and Practice, 30(6), 855-860, DOI: 10.1111/j.15406520.2006.00156.x.

CARTER, D.A., D'SOUZA, F., SIMKINS, B.J. \& SIMPSON, W.G. (2010): "The gender and ethnic diversity of US boards and board committees and firm financial performance", Corporate Governance, 18(5), 396-414, DOI: 10.1111/j.1467-8683.2010.00809.x.

CARTER, D.A., SIMKINS, B.J. \& SIMPSON, W. (2003): "Corporate governance, board diversity and firm value", Financial Review, 38(1), 33-53, DOI: 10.1111/1540-6288.00034.

CASSIMAN, B. \& VEUGELERS, R. (2006): "In search of complementarity in innovation strategy: internal R\&D and external knowledge acquisition", Management Science, 52(1), 68-82, DOI: 10.1287/ mnsc. 1050.0470 .

CATALYST (2004): Census of Women Board Directors of Canada.

CHINCHILLA, N., POELMANS, S. \& LEON, C. (2005): Mujeres directivas bajo el techo de cristal. Directivas en la empresa: criterios de decisión y valores femeninos en la empresa, International Center of Work and Family.

CRUZ, C., JUSTO, R. \& LARRAZA-KINTANA, M. (2018): "When Do Women Make a Better Table? Examining the Influence of Women Directors on Family Firm's Corporate Social Performance", Entrepreneurship Theory and Practice, 43(2), 282-301, DOI: 1177/1042258718796080.

CRUZ, C., LARRAZA-KINTANA, M., GARCÉS-GALDEANO, L. \& BERRONE, P. (2014): "Are family firms really more socially responsible?", Entrepreneurship Theory and Practice, 38(6), 1295-1316, DOI: 10.1111/etap.12125.

DANES, S.M., HABERMAN, H.R. \& MCTAVISH, D. (2005): "Gendered discourse about family business", Family Relations, 54(1), 116-130, DOI: 10.1111/j.0197-6664.2005.00010.x. 
DE MASSIS, A., KOTLAR, J., MAZZOLA, P., MINOLA, T. \& SCIASCIA, S. (2018): "Conflicting Selves: Family Owners' Multiple Goals and Self-Control Agency Problems in Private Firms Show less", Entrepreneurship Theory and Practice, 42(3), 362-389, DOI: 10.1111/etap.12257.

DELOITTE (2017): Women in the boardroom. A global perspective, Global Center for Corporate Governance, Canberra (5th ed).

DEZS, C.L. \& ROSS, D.G. (2011): "Does female representation in top management improve firm performance?", Strategic Management Journal, 33(9), 1072-1089, DOI: 10.1002/smj.1955.

DIÉGUEZ-SOTO, J., MANZANEQUE, M. \& ROJO-RAMÍREZ, A. (2016): "Technological innovation inputs, outputs, and performance: The moderating role of family involvement in management", Family Business Review, 29(3), 327-346, DOI: 10.1177/0894486516646917.

DUMAS, C. (1989): "Understanding of father-daughter and father-son dyads in family-owned businesses", Family Business Review, 2(1), 31-46, DOI: 10.1111/j.1741-6248.1989.00031.x.

DUMAS, C. (1998): "Women's pathways to participation and leadership in the family owned firm", Family Business Review, 11(3), 219-228, DOI: 10.1111/j.1741-6248.1998.00219.x.

ECHEBARRIA-MIGUEL, C. \& LARRAÑAGA-SARRIEGUI, M. (2005): "La igualdad entre mujeres y hombres: una asignatura pendiente", CIRIEC-España, Revista de Economía Pública, Social y Cooperativa, 50, 11-35.

EDLUND, C. (1992): Humanizing the workplace: Incorporating feminine leadership, Public management in an interconnected world (Bailey MT, Mayer RT, eds). Greenwood Press, Westport, CT (US).

ERHARDT, N.L., WERBEL, J.D. \& SHRADER, C.B. (2003): "Board of Director Diversity and Firm Financial Performance", Corporate Governance-An International Review, 11(2), 102-111, DOI: 10.1111/1467-8683.00011.

ESTEBAN-SALVADOR, M.L., GARGALLO-CASTEL, A. \& PÉREZ-SANZ, F.L. (2012), "El papel de la mujer en las cooperativas agrícolas turolenses", CIRIEC-España, Revista de Economía Pública, Social y Cooperativa, 74, 177-205.

ESTEBAN-SALVADOR, M.L., PÉREZ-SANZ, F.L. \& GARGALLO-CASTEL, A. (2017): "Áreas rurales y cooperativas: iniciativas de mujeres para el desarrollo", REVESCO, Revista de Estudios Cooperativos, 127, 116-138, DOI: 10.5209/REVE.58397.

FINKELSTEIN, S. (1992): "Power in top management teams: dimensions, measurement and validation", Academy of Management Journal, 35(3), 505-538, DOI: 10.5465/256485.

GÓMEZ-ÁLVAREZ, J. \& SÁNCHEZ-BARRIOS, M. (2009): "La participación de la mujer en los Consejos de Administración de empresas del Ibex-35", Revista de Humanidades, 16, 105-140.

GÓMEZ-ANSÓN, S. (2005): Diversidad de género en los Consejos de Administración de las sociedades cotizadas y cajas de ahorro españolas, Papeles de la Fundación, 12. 
GORDINI, N. \& RANCATI, E. (2017): "Gender diversity in the Italian boardroom and firm financial performance”, Management Research Review, 40(1), 75-94, DOI: 10.1108/MRR-02-2016-0039.

GUJARATI, D. \& PORTER, D. (2008): Basic Econometrics, McGraw-Hill Education, Boston, MA, US. (5th ed.).

HARRIS, C.K. (2014): "Women directors on public company boards: does a critical mass affect leverage?", Business and Economics Faculty Publications, 10, 139-154.

HAUCK, J. \& PRÜGL, R. (2015): "Innovation activities during intra-family leadership succession in family firms: An empirical study from a socioemotional wealth perspective", Journal of Family Business Strategy, 6(2), 104-118, DOI: 10.1016/j.jfbs.2014.11.002.

HERNÁNDEZ-NICOLÁS, C., MARTÍN-UGEDO, J.F. \& MÍNGUEZ-VERA, A. (2016): "La influencia del género en la dirección de las sociedades cooperativas españolas sobre la rentabilidad y el endeudamiento: un análisis empírico", REVESCO. Revista de Estudios Cooperativos, 122, 135-164, DOI: 10.5209/rev_REVE.2016.v122.52021.

HERNÁNDEZ-ORTIZ, M.J., GARCÍA-MARTí, E., MARTíNEZ-JIMÉNEZ, R., PEDROSA-ORTEGA, C. \& RUIZ-JIMÉNEZ, C. (2020): "El efecto de la diversidad de género sobre el rendimiento de las sociedades cooperativas agroalimentarias españolas", REVESCO. Revista de Estudios Cooperativos, 133, 1-13, DOI: 10.5209/reve.67337.

HIGGS D, 2003. Review of the role and effectiveness of non-executive directors, Non-Executive Directors Review, London, UK.

IEF (2018): Factores de Competitividad y análisis financiero en la Empresa Familiar, Instituto de la Empresa Familiar y Red de Cátedras de Empresa Familiar, Madrid, España.

INFORMA (2013): Las mujeres en los Consejos de Administración y organismos de decisión de las empresas españolas, marzo 2013.

INFORMA (2019): Presencia de las mujeres en la empresa española, marzo 2019.

INMUJERES, (2009): Empresas de mujeres = Empresas exitosas (Recomendaciones para hacer crecer tu negocio), Instituto Nacional de las Mujeres de México, México.

ISOTES (2014): Inspirando: Casos de éxito en diversidad de género.

JIANAKOPLOS, N.A. \& BERNASEK, A. (1998): "Are women more risk averse?", Economic Inquiry, 36(4), 620-630, DOI: 10.1111/j.1465-7295.1998.tb01740.x.

JOECKS, J., PULL, K. \& VETTER, K. (2013): "Gender diversity in the boardroom and firm performance: what exactly constitutes a critical mass?", Journal of Business Ethics, 118(1), 61-72, DOI: 10.1007/s10551-012-1553-6.

JOY, L., CARTER, N., WAGNER, H. \& NARAYANAN, S. (2007): "The bottom line: Corporate performance and women's representation on boards", Catalyst, 1. 
KANTER, R.M. (1977): Men and women of the corporation, Basic Books, Nueva York.

KARRA, N., TRACEY, P. \& PHILLIPS, N. (2006): "Altruism and agency in the family firm: Exploring the role of family, kinship, andethnicity", Entrepreneurship Theory and Practice, 30, 6-861, DOI: 10.1111/j.1540-6520.2006.00157.x.

KOCHAN, T., BEZRUKOVA, K., ELY, R., JACKSON, S., JOSHI, A., JEHN, K., LEONARD, J., LEVINE, D. \& THOMAS, D. (2003): "The effects of diversity on business performance: Report of the diversity research network", Human Resource Management, 42(1), 3-21, DOI: 10.1002/hrm.10061.

KONRAD, A.M., KRAMER, V. \& ERKUT, S. (2008): "Critical mass: the impact of three or more women on corporate boards", Organizational Dynamics, 37(2), 145-164, DOI: 10.1016/j.orgdyn.2008.02.005.

LEACH, P., SMITH, W., HART, A., MORRIS, T., AINSWORTH, J., BETERLSEN, E., IRAQUI, S. \& PASARI, V. (1990): Managing the Family Business in the U.K.: Stoy Hayward Survey in Conjunction with the London Business School, Stoy Harward, London.

LIU, Y., WEI, Z. \& XIE, F. (2014): "Do women directors improve firm performance in China?", Journal of Corporate Finance, 28, 169-184, DOI: 10.1016/j.jcorpfin.2013.11.016.

LÓPEZ-DELGADO, P. \& DIÉGUEZ-SOTO, J. (2018): "Indebtedness in family-managed firms: the moderating role of female directors on the board", Review of Managerial Science, 1-36, DOI: 10.1007/ s11846-018-0307-y.

MAPA (2018): Titularidad compartida de las explotaciones agrarias, enero 2018. Ministerio de Agricultura y Pesca, Alimentación y Medio Ambiente.

MARTÍN-UGEDO, J.F. \& MINGUEZ-VERA, A. (2014): "Firm performance and women on the board: evidence from Spanish small and medium-sized enterprises", Feminist Economics, 20(3), 136162, DOI: 10.1080/13545 701.2014.89540 4.

MARTÍNEZ-JIMÉNEZ, R. (2009): "Research on women in family firms: Current status and future directions", Family Business Review, 22(1), 53-64, DOI: 10.1177/0894486508328813.

MARTíNEZ-ROMERO, M.J., MARTíNEZ-ALONSO, R., CASADO-BELMONTE, M.P. \& ROJO-RAMÍREZ, A. (2019): The moderating effect of family management on $R \& D$ productivity in privately held firms, Entrepreneurship, Innovation and Internationalization (Teixeira NM, Gomes T, Lisboa IM, eds.), Business Strategy and Competitive Advantage (Ed.), Hershey, PA, USA.

MARTíNEZ-ROMERO, M.J. \& ROJO-RAMÍREZ, A.A. (2016): "SEW: Temporal Trajectory and Controversial Issues", European Journal of Family Business, 6(1), 1-9.

MARTíNEZ-ROMERO, M.J. \& ROJO-RAMÍREZ, A.A. (2017): "Socioemotional wealth's implications in the calculus of the minimum rate of return required by family businesses' owners", Review of Managerial Science, 11(1), 95-118, DOI: 10.1007/s11846-015-0181-9. 
MARTÍNEZ-ROMERO, M.J., ROJO-RAMÍREZ, A. \& CASADO-BELMONTE, M.P. (2019): "Value creation in privately held family businesses: The moderating role of socioemotional wealth", Canadian Journal of Administrative Sciences, DOI: 10.1002/cjas.1540.

MICIU (2011): Manual: El género en la investigación, Comunidad Europea.

OAKLEY, J.G. (2000): "Gender-based barriers to senior management positions: Understanding the scarcity of female CEOs”, Journal of Business Ethics, 27(4), 321-334, DOI: 10.1023/A:1006226129868.

ORTIZ, P. \& OLAZ, A.J. (2014): "La mujer en la Empresa Familiar española desde la perspectiva del familiness", Política y Sociedad, 51(2), 481-506, DOI: 10.5209/revPOS0.2014.v51.n2.43647.

PALOMO-ZURDO, R., GUTIÉRREZ-FERNÁNDEZ, M. \& FERNÁNDEZ-TORRES, Y. (2017): "La cuestión del género en los órganos de gobierno de la banca cooperativa", CIRIEC-España, Revista de Economía Pública, Social y Cooperativa, 89, 137-166, DOI: 10.7203/CIRIEC-E.89.8845.

PAVAN, N., RAMACHANDRAN, K., VISHWANATHAN, A. \& CHITTOOR, R. (2018): Standalone family firms lead on gender parity, ISBINSIGHT.

PHILIP, L. \& BHATTACHARYYA, R. (2018): Women directors at family firms face competence versus kinship challenge, The Economic Times.

REGUERA-ALVARADO, N, DE FUENTES, P. \& LAFFARGA, J. (2017): "Does board gender diversity influence financial performance? Evidence from Spain", Journal of Business Ethics, 141(2), 337350, DOI: 10.1007/s10551-015-2735-9.

RODRÍGUEZ-ZAPATERO, M. \& RODRÍGUEZ-JIMÉNEZ, M. (2011): "La mujer en la Empresa Familiar", Revista de Empresa Familiar, 1, 17-26, DOI: 10.5209/rev_POSO.2014.v51.n2.43647.

RODRÍGUEZ-ZAPATERO, M., RODRÍGUEZ-JIMÉNEZ, M. \& RAMÍREZ-FAZ, I. (2013): La mujer en la Empresa Familiar. Una aproximación a la provincia de Córdoba, Servicio de publicaciones de la Universidad de Córdoba, España.

ROSENBLATT, P.C., DE MILK, L., ANDERSON, R.M. \& JOHNSON, P.A. (1985): The family business, Books on Demand, San Francisco, LA, US.

RYAN, M.K. \& HASLAM, S.A. (2005): "The glass cliff: evidence that women are over-representated in precarious leadership positions", British Journal of Management, 16(1), 81-90, DOI: 10.1111/j.1467-8551.2005.00433.x.

SCHEIN, V. \& MUELLER, R. (1992): "Sex role stereotyping and requisite management characteristics: A cross-cultural look", Journal of Organizational Behavior, 13(5), 439-447, DOI: 10.1002/ job.4030130502.

SCHUMACHER, A.J. (2015): Éxito en la sucesión de empresas familiares. factores de influencia, Tesis Doctoral, Universidad de la Rioja, España. 
SCHUMANN, F. (2014): Documental Houshi.

SHRADER, C.B., BLACKBURN, V.B. \& ILES, P. (1997): "Women in management and firm financial performance: an exploratory study", Journal of Managerial Issues, 9(3), 355-372, DOI: 40604152.

SMITH, N., SMITH, V. \& VERNER, M. (2006): "Do women in top management affect firm performance? A panel study of 2,500 Danish firms", International Journal of Performance Management, 55, 569-593, DOI: $10.1108 / 17410400610702160$.

TABACHNICK, B.G. \& FIDELL, L.S. (1996): Using multivariate statics, Harper Collins College Publishers, NY, US. (3rd ed.).

THE FEMALE FTSE REPORT (2004): Cranfield University, School of Management.

TYSON, L. (2003): The Tyson Report on the Recruitment and Development of Non-executive Directors, London Business School, London, UK.

VADNJAL, J. \& ZUPAN, B. (2009): "The role of women in family business", Economic and Business Review, 1(2), 159-177, DOI: 334.012.32:305.

VELANDIA, S.M. \& HERRERA, P.N. (2015): La Empresa Familiar y la mujer imperceptible, XX Congreso Internacional de Contaduría, Administración e Informática, Ciudad Universitaria (México), Oct 7-9.

VANDEKERKHOF, P., STEIJVERS, T., HENDRIKS, W. \& VOORDECKERS, W. (2019): "The effect of nonfamily managers on decision-making quality in family firm TMTs: The role of intra-TMT power asymmetries", Journal of Family Business Strategy, 10(3), 1-1, DOI: 10.1016/j.jfbs.2019.01.002.

VERA, C. \& DEAN, M. (2005): "An examination of the challenges daughters face in family business succession", Family Business Review, 18(4), 321-346, DOI: 10.1111/j.1741-6248.2005.00051.x.

VERSHININA, N., RODGERS, P., MCADAM, M. \& CLINTON, E. (2019): "Transnational migrant entrepreneurship, gender and family business", Global Networks-A Journal of Transnational Affairs, 19(2), 238-260, DOI: 10.1111/glob.12225.

WANG, J. \& COFFEE, B.S. (1992): "Board composition and corporate philanthropy", Journal of Business Ethics, 11(10), 771-778, DOI: 10.1007/BF00872309. 